\title{
BMJ Open Tailored smartphone intervention to promote healthy eating among Brazilian adolescents: a randomised controlled trial protocol
}

\author{
Giselle Rhaisa Melo (1) , ${ }^{1}$ Stefany Correa Lima, ${ }^{1}$ Carolina M dos Santos Chagas, ${ }^{1,2}$ \\ Eduardo Y Nakano, ${ }^{3}$ Natacha Toral ${ }^{1}$
}

To cite: Melo GR, Correa Lima S, M dos Santos Chagas C, et al. Tailored smartphone intervention to promote healthy eating among Brazilian adolescents: a randomised controlled trial protocol. BMJ Open 2020;10:e038896. doi:10.1136/ bmjopen-2020-038896

- Prepublication history for this paper is available online. To view these files, please visit the journal online (http://dx.doi. org/10.1136/bmjopen-2020038896).

Received 27 March 2020 Revised 29 September 2020 Accepted 12 0ctober 2020

Check for updates

(c) Author(s) (or their employer(s)) 2020. Re-use permitted under CC BY-NC. No commercial re-use. See rights and permissions. Published by BMJ.

${ }^{1}$ NESNUT- Department of Nutrition, University of Brasilia, Brasilia, Federal District, Brazil ${ }^{2}$ Department of Nutrition, Federal University of Lavras, Lavras, Minas Gerais, Brazil

${ }^{3}$ Statistics Department, University of Brasília, Brasilia, Federal District, Brazil

Correspondence to Giselle Rhaisa Melo; giselle-mel01502@hotmail.com

\section{ABSTRACT}

Introduction Adolescent eating patterns are

characterised by high consumption of unhealthy foods, which has resulted in an increasing prevalence of overweight and chronic diseases. It is crucial to promote healthy eating habits, and nutritional interventions based on the transtheoretical model have been found to be especially effective. Mobile health strategies also seem promising for adolescents. This study aims to outline a smartphone intervention via WhatsApp for adolescents to promote healthy eating consumption, better nutritional knowledge, self-efficacy in the adoption of healthy practices and progress through the stages of change. Methods and analysis There will be three distinct groups in this randomised study: a general intervention group (GG), in which the participants will receive the same healthy eating messages, based on the Brazilian food guide; a tailored intervention group (TG), in which the participants will receive healthy eating messages based on their stage of change; and a control group (CG), in which participants will receive messages on a different theme. Possession of a smartphone, use of WhatsApp and being a senior student (16-19 years) from a public school of the Federal District of Brazil will be the study's inclusion criteria. Rural schools will be excluded. The sample size estimated is 390 individuals: 38 in the GG, 314 in the TG and 38 in the CG. The intervention will last 6 weeks, with a daily message sent to the students. We will investigate nutritional knowledge, self-efficacy in the adoption of healthy eating practices, food consumption and stages of change using preintervention and postintervention questionnaires. Memorisation of the messages will be also assessed.

Ethics and dissemination The study was approved by the University of Brasília, School of Health Sciences and Research Ethics Committee. At the end of the study, the participating schools will receive a printed report with the main results of the intervention.

Trial registration number RBR-5b9jk7.

\section{INTRODUCTION}

The eating patterns of adolescents are characterised by high sugar, fat and sodium consumption, and low fruit and vegetable consumption. Data from the 21 countries
Strengths and limitations of this study

- The present nutritional intervention was planned according to the study population (adolescents) in all its facets (the format, length, periodicity, timing and language of the messages).

- All participants will receive some type of educational health promotion messages on their own smartphones, using WhatsApp, a free and widely accepted messaging service, as the delivery platform.

- The healthy eating messages are based on the Dietary Guidelines for the Brazilian Population and have been previously validated.

- One of the study's aims is to compare the effects of general messages about healthy eating with messages specifically tailored to the participant's stage of change.

- A limitation of the study is its inability to determine if the messages will be read in their entirety.

involved in the WHO's STEPwise Approach to Surveillance show that only $10 \%$ of $15-19$ year olds meet the daily recommendations for fruit and vegetable consumption. ${ }^{1}$ The situation in Brazil is no different: data from a national study revealed low fruit and vegetable consumption, and high ultra-processed food consumption in both sexes. ${ }^{2}$

Such inadequate consumption corroborates the high incidence and prevalence of overweight and chronic diseases currently found among adolescents. Projections indicate that by 2022 , the overweight/obesity rate will be higher among 5-19year olds than the rate of underweight. ${ }^{3}$ In Brazil, the obesity and overweight rates among Brazilian adolescents are $8.4 \%$ and $17.1 \%$, respectively. ${ }^{45}$

Excess weight, although not the sole explanatory factor, influences the onset of chronic non-communicable diseases, metabolic diseases and so on, and is thus quite harmful to the overall health of adolescents. ${ }^{6}$ Thus, it is crucial to promote healthy eating and 
a healthy environment to prevent diseases, and ensure food and nutritional security at this stage of development. A recent systematic review pointed out that nutritional interventions based on theoretical models are more effective in establishing new health behaviours among adolescents. ${ }^{7}$ The transtheoretical model stands out as a way of enhancing the effects of behavioural interventions. This theory proposes that behavioural change occurs over time and is mediated by four dimensions: stages of change, processes of change, self-efficacy and decisional balance.

The five stages of change (precontemplation, contemplation, decision, action and maintenance) are the central components of the model. They correspond to the temporal dimension of the change, which influences treatment adherence. ${ }^{8}$ Regarding nutritional behaviour, each stage represents different perceptions and degrees of motivation to modify one's diet. They include precontemplation (no intention to change in the near future), contemplation (beginning to consider change, but many barriers are identified), decision (preparation and confidence to change), action (visible and concrete, although recent, diet changes) and maintenance (diet changes sustained for a more significant period of time, usually more than 6 months) ${ }^{9}$

According to the transtheoretical model, processes of change and self-efficacy are the activities and cognitions by which people progress through the stages of change. ${ }^{9}$ Ten processes of change can be used to facilitate progress from early stage to later stages, and each is specific to the transition from one stage to another. When moving between each stage, self-efficacy, that is, the confidence to handle situations without relapsing to former behaviour, is also expected to increase. ${ }^{9}$

Thus, people should respond to and benefit more from tailored interventions whose content is directed at their stage of change. A systematic review by Lee $e t a l^{8}$ of nursing interventions used for diet, physical activity and smoking cessation management found that the majority of the stage-matched interventions were more effective than non-stage-matched interventions. Another systematic review found that interventions aimed at specific stages of change led to lower fat intake, and higher fruit and vegetable consumption. ${ }^{10}$ It is also emphasised that most of the selected interventions used the internet and/ or mobile phones.

The role of technological tools in nutritional interventions should also be mentioned. The term 'digital health' can be defined as the use of technology to promote/ maintain health or prevent/treat disease, and includes tools such as mobile health (mHealth) ${ }^{11}{ }^{12}$ In Brazil, the age group with the highest percentage of internet users is $20-24$ year olds $(88.4 \%)$, followed by $18-19$ year olds $(88.1 \%)$, regardless of socioeconomic status. Among the devices used to access the internet for private use, the smartphone is still the most common (98.7\%), and the main purpose of network access is to send or receive text, voice or picture messages by applications other than email. ${ }^{13}$
One strategy for using smartphones in interventions is to send health-related messages. The advantage of mHealth messaging interventions is that they involve sending and collecting data in real time. ${ }^{14}$ The widespread success of WhatsApp, currently the most popular free messaging service, could be due to the fact that only a mobile internet connection (data plan or Wi-Fi) is required to use it. ${ }^{15}$

Although some studies have reported using WhatsApp in healthcare settings, ${ }^{16-18}$ only one, to the best of our knowledge, has used it in smartphone interventions with adolescents. This study, conducted in Brazil, used WhatsApp as a complementary intervention strategy with a sample of adolescent girls from low-income communities. Significant intervention effects for waist circumference, dietary intake and leisure-time sedentary behaviours were reported. ${ }^{19}$ Thus, further studies on the efficacy of WhatsApp as an intervention tool should be conducted.

This study aims to outline smartphone intervention for adolescents to promote healthy eating via WhatsApp, comparing general healthy eating promotion messages with messages tailored to each participant's stage of change.

\section{METHODS AND ANALYSIS \\ Study design}

This is a protocol of a randomised study in which a nutritional intervention will be held via WhatsApp among adolescents in public schools in the Federal District of Brazil. The intervention groups will receive messages about healthy eating, according to the Dietary Guidelines for the Brazilian Population. ${ }^{20}$ The transtheoretical model will be used as a tool for tailoring the messages sent to one of the intervention groups. This study follows the Standard Protocol Items: Recommendations for Interventional Trials guidelines. ${ }^{21}$

\section{Participants and eligibility criteria}

The study will be conducted with senior students in public high schools that participate in the Health in School Program, which includes 19 schools and 4183 adolescents. This programme is a part of a national effort involving the Brazilian Ministry of Health and Education. It is aimed at public schools, which generally include lower and middle income students. ${ }^{22}$ Schools linked with this programme were chosen in hopes of finding greater openness to health educational interventions, and high school seniors were chosen due to the greater likelihood of having a smartphone and internet access. Possession of a smartphone and use of WhatsApp will be the study's inclusion criteria. Schools in rural areas and night school students will be excluded for logistical reasons.

\section{Intervention}

During the intervention, all participants will be sent short WhatsApp messages daily at lunchtime. The researchers will be instructed not to interact with the students. A 
total of 42 messages will be sent, one per day, including three orientation messages (one welcoming the participants to the study, one indicating that the study is halfway complete and one at the conclusion of the study, thanking the students for their participation) and 39 thematic messages. The intervention will last 42 days $(6$ weeks). Since participants will not be required to change their WhatsApp settings to participate in the study, the percentage of participants who will read the messages cannot be analysed.

\section{Groups and message content}

Participants will be allocated into three groups. The general intervention group (GG) will receive one healthy eating message per day for 6 weeks. These messages were selected from a previous message validation study in adults based on the above-mentioned dietary guidelines, ${ }^{20}$ and their language and content were adapted by a panel of experts to better communicate with youth culture. The messages were then validated with a young, nationally representative population from all regions of Brazil. These short, direct messages use both colloquial (ie, including personal pronouns) and suggestive language, based on a previously described model for developing educational messages for adolescents. ${ }^{23}$

The tailored intervention group (TG), will receive one tailored healthy eating message per day for 6 weeks. The messages will be tailored to each stage of change. ${ }^{9}$ An algorithm used in a previous study ${ }^{24}$ was translated to Portuguese and will identify each participant's stage at baseline. The TG will be divided into three subgroups: (1) precontemplation and contemplation, (2) decision and (3) action and maintenance, a model used in previous nutritional interventions. ${ }^{10}$
The messages for the TG were based on those of the GG but considered the process of change, which is expected to increase participant motivation and encourage progress through the stages. ${ }^{9}$ For subgroup 1 , the modifications were based on raising consciousness, dramatic relief and environmental re-evaluation processes. For subgroup 2 , they were based on self-re-evaluation, self-liberation and social liberation. Finally, for subgroup 3, they were based on counter-conditioning, helping relationships, and reinforcement management and stimulus control. Thus, each general message was adapted into three messages, one for each subgroup, except for the five concept messages used in the Brazilian Dietary Guidelines. ${ }^{20}$

The final 34 messages were developed by three experienced researchers (R1, R2 and R3) in healthy eating, nutrition and the transtheoretical model. The messages were initially developed by $\mathrm{R} 1$, revised by $\mathrm{R} 2$ and revised again, in a face-to-face meeting, by R1, R2 and R3. The messages were validated through an online questionnaire with two research groups from different regions of Brazil, whose goals were to determine: (1) whether the modified messages for each group contained the same content as the original ones; and (2) whether the involved process of change actually fit the version of the message. Finally, analysis of the validation responses and editing the messages was performed by three expert panels with six invited researchers per panel. Figure 1 shows an example of a message adaptation for the tailored group.

Based on the attachment theory, which explains that a positive parent-child relationship provides a safe basis for engaging in healthy future relationships, ${ }^{25}$ the control group (CG) will receive one message per day for 6 weeks about the prevention of dating violence, based on a

\section{STAGE OF CHANGE SUBGROUPS}

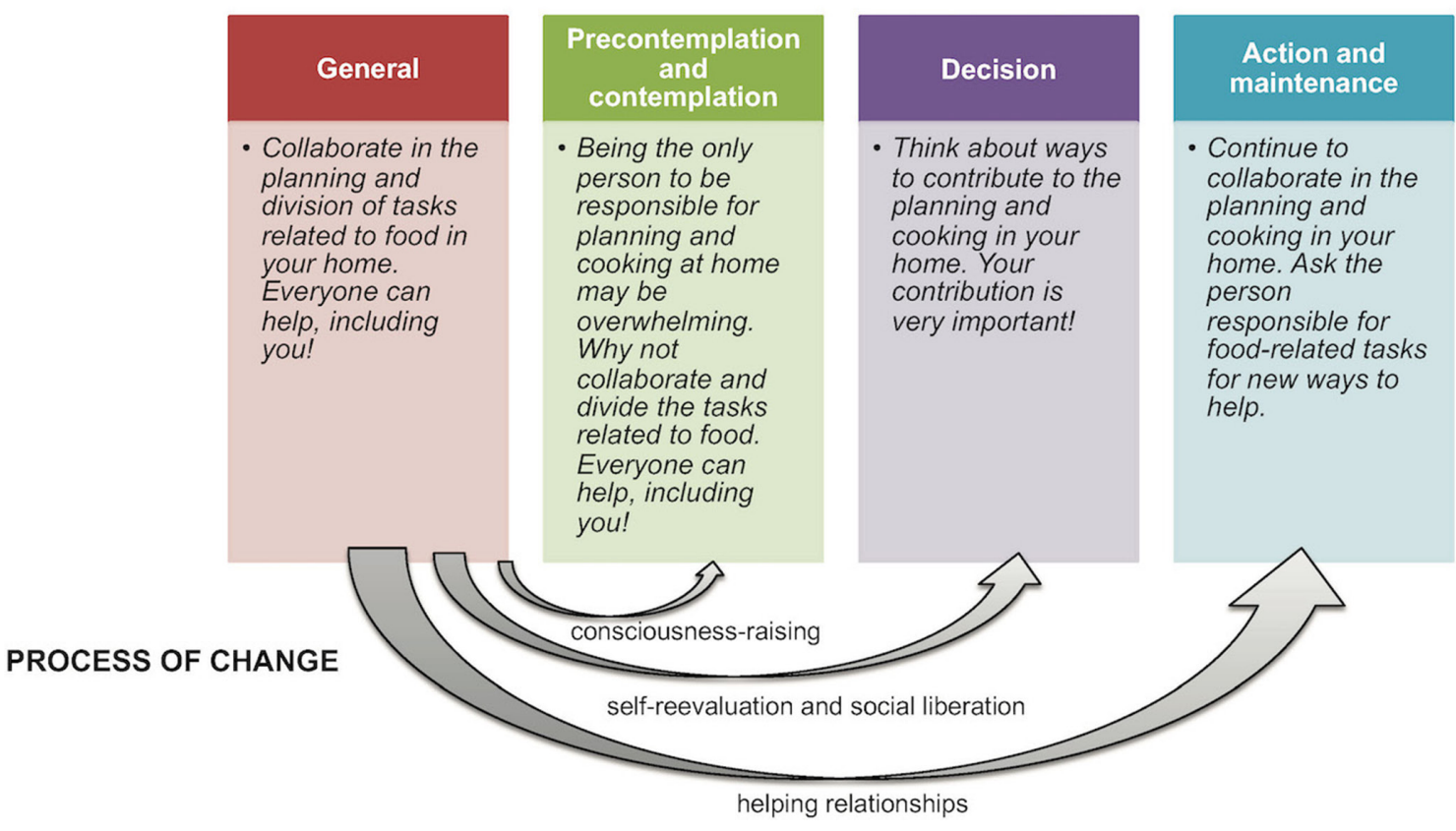

Figure 1 Example of a message adaptation for the tailored group. 
pre-existing programme designed for adolescents. To counteract the Hawthorne effect, these messages involve no healthy eating content. ${ }^{26}$

\section{Sample size and recruitment}

Sample size calculation was based on expected self-efficacy in the adoption of healthy practices during the intervention period, considering a $15 \%$ increase in self-efficacy scores clinically significant, an SD of $4.79,{ }^{27}$ a significance level of $5 \%$ and a power of $80 \%$. The distribution of stages of change observed in the literature ${ }^{28}$ was also considered: $68 \%, 12 \%$ and $20 \%$ in precontemplation/ contemplation, decision and action/maintenance stages, respectively. For such conditions, the minimum sample in the smallest intervention subgroup (decision) should be 26 adolescents.

In addition, when calculating the minimum sample, an estimated loss of $31.8 \%$ was added between preintervention and postintervention. ${ }^{29}$ Thus, the minimum sample was 390 individuals: 38 in the CG, 38 in the GG and 314 in the TG.

A computer-generated randomisation method, supervised by a statistician, will be used to allocate schools in each group, with new schools drawn from the pool until the minimum sample is reached. Schools will be drawn for groups in the following order: CG, GG and TG. School recruitment will occur through personal visits to the principals. If a school denies participation, a new school will be drawn from the pool to replace the previously allocated school. The researchers will present the project to the students in the classroom, and those who are interested in participating must return with the consent form signed by themselves as well as by their parents or legal guardians within 1 week. Figure 2 shows the flowchart of recruitment and intervention of the study.

\section{Measures}

All participants will complete a preintervention and postintervention self-administered questionnaire on a regular school day. The following variables will be investigated: age, gender, maternal education, food consumption, stage of change, nutritional knowledge and self-efficacy in the adoption of healthy practices. Maternal education will be assessed through multiple questions used in national population surveys. ${ }^{30}$ To assess food consumption, a food frequency questionnaire will be applied that includes markers of healthy and unhealthy eating (especially among adolescents), based on previous

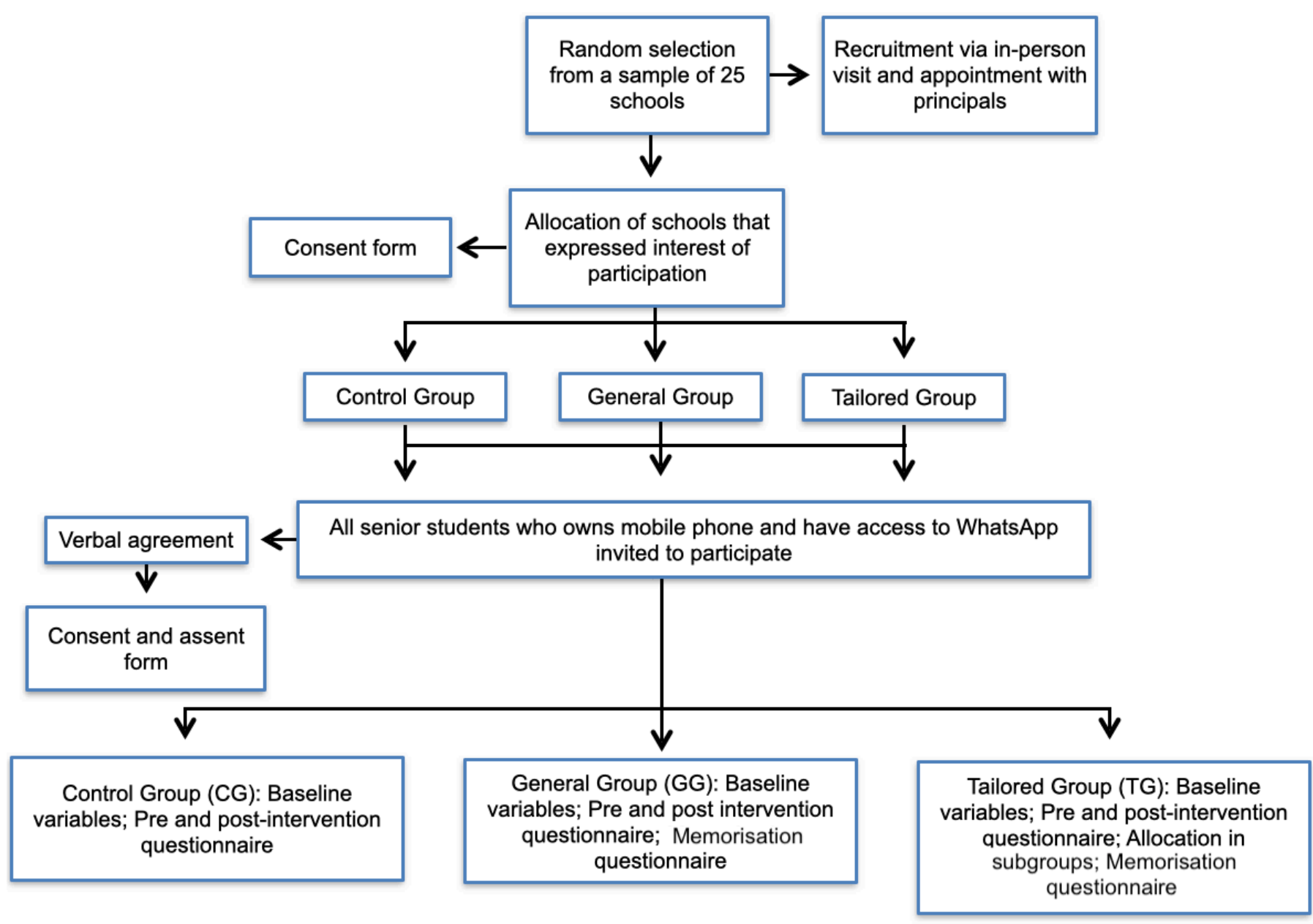

Figure 2 Study procedure flow diagram. 
national surveys. ${ }^{30} 31$ Participants will be asked if they ate that particular food item the day before, responding with either yes or no.

The stages of change toward a healthy diet will be evaluated. An algorithm, validated in a previous Spanish study on adolescents and translated into Portuguese, ${ }^{24}$ will be used to identify the respondent's stage based on his/her food perceptions. Respondents who have never pursued healthy eating habits will be asked if they have considered changing their diet in the last month: negative responses will be classified as precontemplation and positive ones as contemplation or decision, depending on the respondents' degree of confidence that they will succeed in making their diet healthier in the next month. Regarding those who are currently trying and/or have tried to maintain a healthy diet: if they began at least 6 months ago, they will be classified as maintenance, but if it has been less than 6 months, they will be classified as action stage.

To assess nutritional knowledge and self-efficacy in the adoption of healthy practices, an instrument developed and validated for nutritional interventions with Brazilian adolescents will be used. ${ }^{32}$ For the nutritional knowledge module, the adolescents will evaluate each statement on a Likert scale ranging from 1 ('I don't agree at all') to 5 ('I totally agree'); for the self-efficacy module, the scale will range from 1 ('Definitely no') to 5 ('Definitely yes').

The food frequency questionnaire and the scales used to assess nutritional knowledge and self-efficacy in the adoption of healthy practices will generate item ratings that will be summed to yield a total score. Higher scores for healthy eating markers and lower scores for unhealthy eating markers will indicate a better diet quality. Higher scores for nutritional knowledge and self-efficacy will indicate improvement in these variables. The final version of the study questionnaire translated to English can be found in the online supplemental material.

\section{Pilot test of the questionnaire and preliminary qualitative research}

To develop an intervention more suited to the target audience, three focus groups were conducted with a convenience sample of 34 students from a public school not involved in the main study, although the students were from the same grade and region as the participants of the main study. A semi-structured script with questions divided into three blocks was used to evaluate (1) the best platform for receiving messages about healthy eating (a new app, WhatsApp or SMS); (2) the most appropriate message format (short or long; text only or text with images/videos); (3) the most convenient time of the day (morning, afternoon or evening) and frequency (daily or every other day) to receive messages.

The adolescents showed greater interest in WhatsApp due to its ease of use and its presence in their daily routines. Short objective text messages were preferred; long messages were considered tiring and were unlikely to be read in their entirety. The students preferred receiving messages daily at less busy times, such as before school or at lunchtime.

The final version of the questionnaire was also evaluated in a pilot study at the same school in which the focus groups were conducted. On average, the students took $5-10 \mathrm{~min}$ to complete it and only one item in the knowledge module needed rewording due to reasons of ambiguity. No doubts were expressed about the other items and statements. The intervention itself was not evaluated in this pilot test.

\section{Message memorisation}

In a subsample of GG and TG, memorisation of the healthy eating messages will be assessed through a questionnaire based on a previously tested instrument for measuring content memorisation. ${ }^{33}$ This step will occur approximately 1 month after the end of the intervention.

The participants will initially be asked to recite three messages they currently remember. The five messages involving the main concepts from the Brazilian Dietary Guidelines ${ }^{20}$ will be shown and the participants will be asked how well they remember each message according to a 5-point Likert scale, ranging from 1 ('I remember that message well') to 5 ('I don't remember that message at all'). In this case, item ratings will also be summed to yield a total score, with higher scores indicating better long-term memorisation.

\section{Data analysis}

Kobo Toolbox, a programme designed for field data collection, will be used to record the questionnaire responses. To accomplish this, a form with limited response options (to minimise typing errors) will be developed, and each participant will be given an individual identification number to protect confidentiality. The data will initially be analysed descriptively as means, medians, SDs and frequencies. Age, gender and maternal education will be used to describe the study population at baseline.

The intervention's impact on food consumption, nutritional knowledge, self-efficacy in the adoption of healthy practices and stages of change will be assessed through two-way mixed analysis of covariance (ANCOVA) with repeated measures with group as between-subject factor, time (preintervention and postintervention) as the withinsubject factor and potential confounders as covariates. The statistical analysis will be performed in SPSS V.20.0, with a significance level of $5 \%$. Message memorisation will be analysed descriptively through means, medians, SDs and frequencies, and one-way ANCOVA followed by Bonferroni post-hoc tests will be used to determine the degree of memorisation for each message.

\section{Participant and public involvement}

The intervention components (the platform, format, number and type of messages) were designed around the participants' preferences. No participants will be involved in recruitment for/or conducting this study. Although individual data will not be disseminated to participants, 
each participating school will receive a printed report with the main results of the intervention.

\section{Hypothesis}

Our hypothesis is that adolescents who participate in the intervention tailored to their specific stage of change will achieve better results than the CG or the GG, such as increased healthy food consumption and reduced unhealthy food consumption, greater nutritional knowledge and self-efficacy in the adoption of healthy practices, and progressing through the stages of change. It is also expected that the general (non-tailored) intervention will produce better results than the CG.

\section{ETHICS AND DISSEMINATION}

The study was approved by the University of Brasília, School of Health Sciences and Research Ethics Committee (no. 90326218.6.0000.0030). The schools and students, as well as their parents or legal guardians, will provide written consent prior to participation. Also, on completion of the study, the CG will receive the same healthy eating material that was provided to the GG.

The data will be released through a publication. At the end of the study, the participating schools, as well as the Brazilian Ministry of Health and the National Council for Scientific and Technological Development, will receive a printed report with the main results of the intervention, although they will not interfere with the data analysis or publication. No individual information of enrolled participants will be shared to protect confidentiality.

Contributors GRM, SCL, CMdSC and NT made substantial contributions to the study's conception and design. NT obtained funding. GRM, SCL, EYN and NT drafted the manuscript. EYN was involved in all statistical components, as well as in the randomisation process. All authors approved the final version of the manuscript.

Funding This study was funded by the National Council for Scientific and Technological Development - CNPq (CNPq/MS/SCTIE/DECIT/SAS/DAB/CGAN Pesquisas em Alimentação e Nutrição n.13/2017, process number 408386/20177), but it was not involved in the study design or the collection, analysis or interpretation of the data.

Competing interests None declared.

Patient consent for publication Not required.

Provenance and peer review Not commissioned; externally peer-reviewed.

Supplemental material This content has been supplied by the author(s). It has not been vetted by BMJ Publishing Group Limited (BMJ) and may not have been peer-reviewed. Any opinions or recommendations discussed are solely those of the author(s) and are not endorsed by BMJ. BMJ disclaims all liability and responsibility arising from any reliance placed on the content. Where the content includes any translated material, BMJ does not warrant the accuracy and reliability of the translations (including but not limited to local regulations, clinical guidelines, terminology, drug names and drug dosages), and is not responsible for any error and/or omissions arising from translation and adaptation or otherwise.

Open access This is an open access article distributed in accordance with the Creative Commons Attribution Non Commercial (CC BY-NC 4.0) license, which permits others to distribute, remix, adapt, build upon this work non-commercially, and license their derivative works on different terms, provided the original work is properly cited, appropriate credit is given, any changes made indicated, and the use is non-commercial. See: http://creativecommons.org/licenses/by-nc/4.0/.

\section{ORCID iD}

Giselle Rhaisa Melo http://orcid.org/0000-0003-4918-8377
REFERENCES

1 Food and Agriculture Organization of the United Nations (FAO). Rome 2015. fruit and vegetables for health. Available: http://www. who.int/dietphysicalactivity/publications/fruit_vegetables_report.pdf

2 Souza AdeM, Barufaldi LA, Abreu GdeA, De Moura Souza A, De Azevedo Abreu G, et al. ERICA: intake of macro and micronutrients of Brazilian adolescents. Rev Saude Publica 2016;50 Suppl 1:1s-15.

3 World Health Organization (WHO). Tenfold increase in childhood and adolescent obesity in four decades: new study by Imperial College London and WHO. Saudi Med J 2017;38:1162.

4 Bloch KV, Cardoso MA, Sichieri R. Study of cardiovascular risk factors in adolescents (ERICA): results and potentiality. Rev Saude Publica 2016;50 Suppl 1:3-5.

5 Costa CDS, Flores TR, Wendt A, et al. Sedentary behavior and consumption of ultra-processed foods by Brazilian adolescents: Brazilian national school health survey (PeNSE), 2015. Cad Saude Publica 2018;34:1-11.

6 Oliveira JS, Barufaldi LA, Abreu GdeA, De Azevedo Abreu G, et al. ERICA: use of screens and consumption of meals and snacks by Brazilian adolescents. Rev Saude Publica 2016;50 Suppl 1:1s-9.

7 do Amaral E Melo GR, de Carvalho Silva Vargas F, Dos Santos Chagas CM, et al. Nutritional interventions for adolescents using information and communication technologies (ICTs): a systematic review. PLoS One 2017;12:1-7.

8 Lee JY, Park H-A, Min YH. Transtheoretical model-based nursing intervention on lifestyle change: a review focused on intervention delivery methods. Asian Nurs Res 2015;9:158-67.

9 Prochaska JO, DiClemente CC, Norcross JC. In search of how people change. applications to addictive behaviors. Am Psychol 1992;47:1102-14.

10 Carvalho de Menezes M, Bedeschi LB, Santos LCD, et al. Interventions directed at eating habits and physical activity using the Transtheoretical model: a systematic review. Nutr Hosp 2016;33:1194-204.

11 Kumar S, Nilsen WJ, Abernethy A, et al. Mobile health technology evaluation: the mHealth evidence workshop. Am J Prev Med 2013;45:228-36.

12 Ippoliti NB, L'Engle K, L'Engle K. Meet us on the phone: mobile phone programs for adolescent sexual and reproductive health in low-to-middle income countries. Reprod Health 2017;14:11.

13 Instituto Brasileiro de Geografia e Estatística (IBGE). Pesquisa Nacional por Amostra de Domicílios: Acesso Internet e Televisão e Posse de Telefone Móvel Celular para Uso, 2018. Available: https:// biblioteca.ibge.gov.br/visualizacao/livros/liv101631_informativo.pdf

14 Devine KA, Viola AS, Coups EJ, et al. Digital health interventions for adolescent and young adult cancer survivors. JCO Clin Cancer Inform 2018;2:1-15.

15 Montag C, Błaszkiewicz K, Sariyska R, et al. Smartphone usage in the 21st century: who is active on WhatsApp? BMC Res Notes 2015;8:4-9.

16 Giordano V, Koch H, Godoy-Santos A, et al. WhatsApp messenger as an adjunctive tool for telemedicine: an overview. Interact $J$ Med Res 2017;6:e11.

17 Döğer E, Bozbulut R, Soysal Acar A. Şebnem, ŞebnemSoysalAcar $A$, et al. Effect of telehealth system on glycemic control in children and adolescents with type 1 diabetes. J Clin Res Pediatr Endocrinol 2019;11:70-5.

18 Coleman E, O'Connor E. The role of WhatsApp $\AA$ in medical education; a scoping review and instructional design model. BMC Med Educ 2019;19:279.

19 Leme ACB, Lubans DR, Guerra PH, et al. Preventing obesity among Brazilian adolescent girls: six-month outcomes of the healthy habits, healthy Girls-Brazil school-based randomized controlled trial. Prev Med 2016:86:77-83.

20 Brazil. Ministry of Health. Secretariat of Health Care. Primary Health Care Department. Dietary Guidelines fo the Brazilian Population. 2 ed. Brasília-DF, Ministry of Health, 2015. Available: http://bvsms. saude.gov.br/bvs/publicacoes/dietary_guidelines_brazilian_ population.pdf

21 Schulz KF, Grimes DA. Get in the spirit with SPIRIT 2013: protocol content guideline for clinical trials. Contraception 2013;88:676-7.

22 Brasil EGM, Silva RMda, Silva MRFda, et al. Adolescent health promotion and the School Health Program: complexity in the articulation of health and education. Rev Esc Enferm USP 2017;51:e03276.

23 Hingle M, Yoon D, Fowler J, et al. Collection and visualization of dietary behavior and reasons for eating using Twitter. J Med Internet Res 2013;15:1-17.

24 López-Azpiazu I, Martinez-González MA, León-Mateos A, et al. Stages of dietary change and nutrition attitudes in the Spanish population. Public Health 2000;114:183-9. 
25 Bretherton I. The origins of attachment theory: John Bowlby and Mary Ainsworth. Dev Psychol 1992;28:759-75.

26 McCarney R, Warner J, Iliffe S, et al. The Hawthorne effect: a randomised, controlled trial. BMC Med Res Methodol 2007;7:1-8.

27 Wall DE, Least C, Gromis J, et al. Nutrition education intervention improves vegetable-related attitude, self-efficacy, preference, and knowledge of fourth-grade students. J Sch Health 2012;82:37-43.

28 Cunha DB, de Souza BdaSN, da Veiga GV, et al. Readiness for behavioral change and variation in food consumption among adolescents from a school-based community trial in Duque de Caxias, RJ. Rev Bras Epidemiol 2015;18:655-65.

29 Fonseca LG, Bertolin MNT, Gubert MB, et al. Effects of a nutritional intervention using pictorial representations for promoting knowledge and practices of healthy eating among Brazilian adolescents. PLOS One 2019;14:e0213277.
30 Instituto Brasileiro de Geografia e Estatística (IBGE). Ministério do Planejamento, Orçamento E Gestão. Pesquisa Nacional POR Amostra de Domicílios, 2013. Available: https://biblioteca.ibge.gov. br/visualizacao/periodicos/59/pnad 2013 v33 br.pdf

31 Souza AdeM, Pereira RA, Yokoo EM, et al. Most consumed foods in Brazil: national dietary survey 2008-2009. Rev Saude Publica 2013;47 Suppl 1:190-9.

32 Chagas CMdosS, Melo GR-S, Botelho RBA, et al. Effects of the Rango Cards game intervention on food consumption, nutritional knowledge and self-efficacy in the adoption of healthy eating practices of high school students: a cluster randomised controlled trial. Public Health Nutr 2020;23:2424-33.

33 Micali FG, Diez-Garcia RW. Pictorial instrument of food and nutrition education for promoting healthy eating. Rev Nutr 2016;29:917-28. 